\title{
THE CONSERVATIVE TREATMENT OF THE TENSION LESIONS OF STAPHYLOCOCCAL PNEUMONIA IN INFANCY \\ BY
}

\author{
JAMES S. DAVIDSON \\ From the Royal Infirmary, Bradford
}

(RECEIVED FOR PUBLICATION APRIL 4, 1960)

Characteristic lesions of staphylococcal pneumonia in infancy are the tension cyst of lung and tension pyopneumothorax. The radiological appearances are often alarming and, at first sight, seem to indicate the necessity for urgent aspiration or drainage. The two obvious dangers are those of immediate suffocation (the French refer to the tension cysts as bulles suffocantes) and of gross pleural thickening as a later complication. The dramatic radiological appearances, the fear that the infant will suffocate, or the expectation of late morbidity may result in unnecessary surgical intervention. The object of this paper is to describe how the size of the pneumatocele or pneumothorax may be controlled, and to show how tension within the lesion during the phase of active infection tends to keep the air space empty and to prevent the deposition of fibrin.

To persevere with conservative treatment in a desperately ill baby whose radiographs show a large pneumatocele or pneumothorax is not easy. Confidence in the method is essential. A striking feature of most of the published series is the infrequency with which suffocation is, in fact, a cause of death. In a series of complications of staphylococcal pneumonia described by Sabiston, Hopkins, Cooke, and Bennett (1959), catheter drainage was used in $43 \%$ of patients with empyema of whom $23 \%$ had associated pneumothorax, but they make the following comment:

"An analysis of the 12 deaths that occurred in the 67 cases reported indicates that fulminating infections or other complicating disease, rather than recognizable surgical complications, were important in the outcome."

Consideration of the cause of death in four of my own series of 24 infants points to the same conclusion :

(1) A baby, 5 weeks old on admission, died a month later. The radiographs were unusual in that they showed emphysema only, sometimes in one lobe and sometimes in another. At necropsy, both upper lobes were emphysematous. Both lower lobes and the middle lobe were solid and contained multiple small staphylococcal abscesses.

(2) Death occurred a few hours after admission in a baby aged 10 months. Necropsy showed staphylococcal meningitis and pericarditis and multiple foci of staphylococcal infection throughout both lungs.

(3) The third fatality was in a 5-week-old baby. Initially there was a pneumothorax with consolidation and cavitation of the right lung. The right upper lobe expanded spontaneously as the pneumothorax disappeared. At post-mortem examination many small staphylococcal abscesses were found throughout the right lung.

(4) Death occurred in a 7-month-old baby who, at necropsy, was found to have staphylococcal infection of both lungs with a small thin-walled cyst in the left lung. This baby had, in addition, fibrocystic disease of the pancreas.

It is difficult to see how surgical intervention could have saved any one of these babies.

As to morbidity, it must be rare to see an older child or adolescent with a frozen chest or gross bronchiectasis that can certainly be traced back to a staphylococcal pneumonia in infancy. The mortality has been sufficiently low for some years for such cases to exist in recognizable numbers if late complications were greatly to be feared.

With such points in mind, one may undertake conservative management with some confidence.

\section{RADIOGRAPHIC APPEARANCES}

A few typical radiographs will serve to show the progress of tension cysts and tension pyopneumothorax without surgical intervention.

For an air space of moderate size (Fig. 1a), conservative treatment is adequate if the space is prevented from enlarging. The space disappears spontaneously and the radiographs become normal (Fig. 1b). 
The following three cases have more alarming radiographs.

(1) A baby, aged 5 months, developed a moderately large air space on the left side with some mediastinal shift (Fig. 2a). The space was empty and remained so. Two months later, under conservative treatment, the radiographs had returned to normal (Fig. 2b).

(2) A pyopneumothorax with mediastinal displacement occurred in a 9-month-old baby (Fig. 3a). Six days later, the volume of fluid had decreased spontaneously (Fig. 3b). A radiograph taken a month later (Fig. 3c) showed return to normal without aspiration or drainage.

(3) A 3-month-old baby developed a pyopneumothorax with mediastinal displacement (Fig.
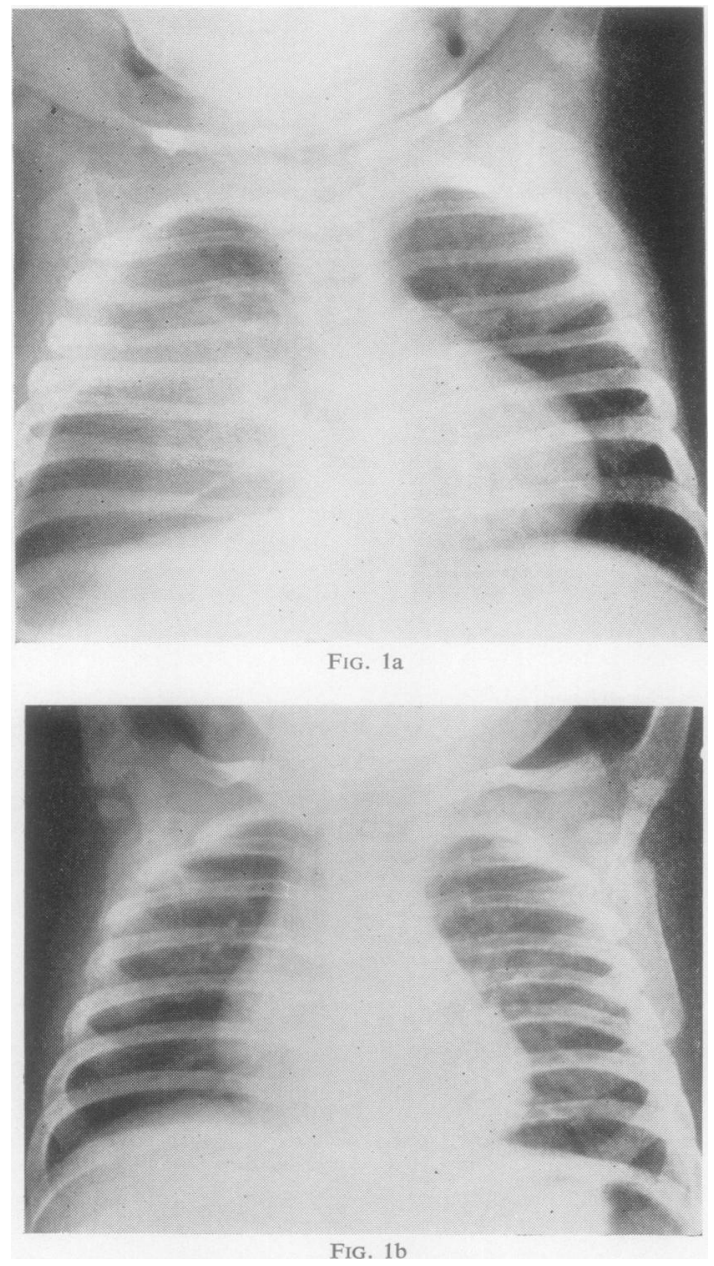

FIG. 1 ( $a$ and $b$ ).-Films showing the progress of an air space of moderate size over one month. 4a). The fluid disappeared spontaneously leaving $\underset{\vec{*}}{\vec{\rho}}$ the characteristic empty space (Fig. 4b). Subse- $\frac{-}{0}$ quently the radiographic appearances returned to $\frac{D}{5}$ normal.

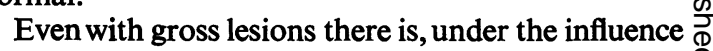
of antibiotics, a tendency towards complete clinical $\stackrel{\circ}{\circ}$ and radiological cure. The tension space is often $\infty$ empty when first seen. If so, it remains empty. If $\vec{O}$ it contains fluid to begin with, the fluid tends to $-\overrightarrow{-}$ disappear without aspiration or drainage.

The same tendency towards spontaneous emptying $\stackrel{\rho}{\vec{f}}$ is seen in pneumatoceles. Initially, a tension cyst $x$
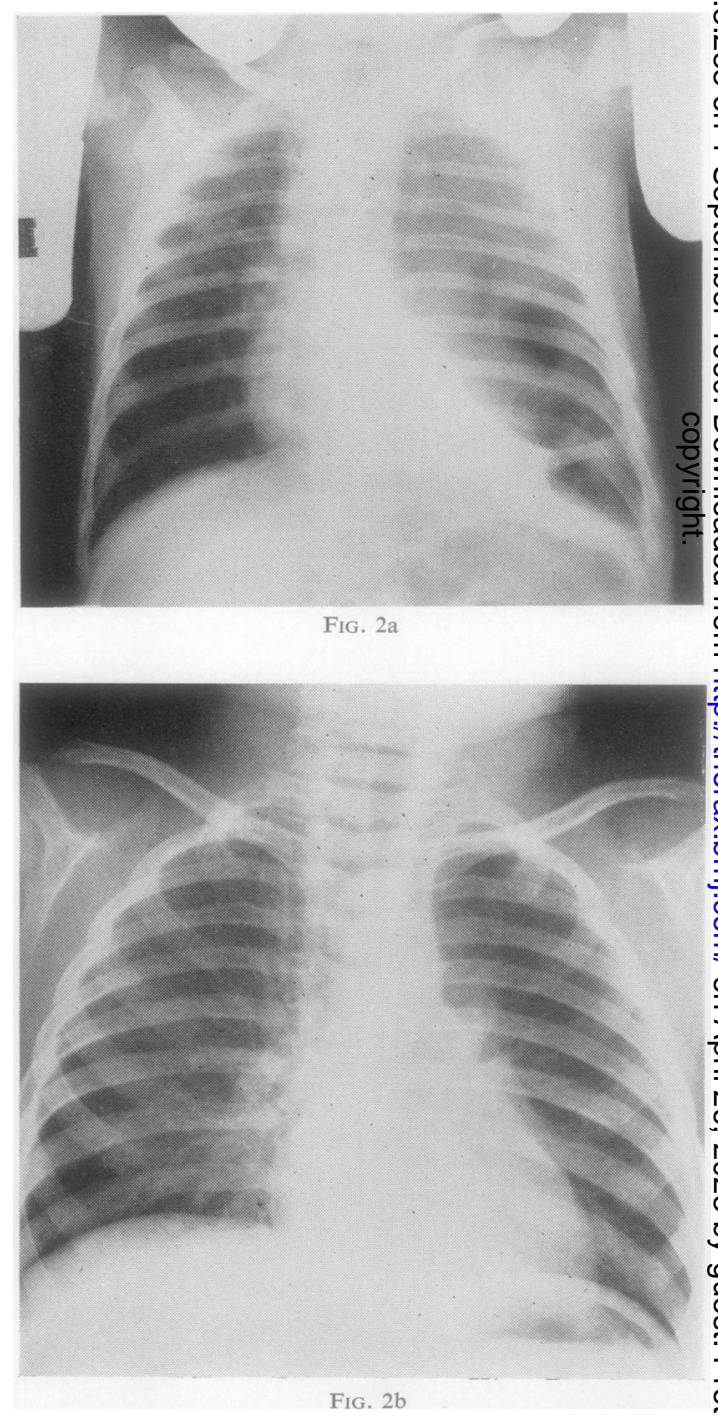

FIG. 2 ( $a$ and $b$ ).-Radiographs showing the progress of a large air space in a baby aged 5 months over two months. 
may contain fluid (Fig. 5a). It is sometimes described as a pneumatocele filling up with fluid. It is more correctly described as a pneumatocele emptying itself of fluid. It is important to recognize that the space increases in size as it empties. As long as the tension in it is maintained, the enlarging pneumatocele becomes empty (Fig. 5b).

The classical lung abscess decreases in size concentrically as it drains via the bronchus. The pneumatocele, on the other hand, is a tension lesion. If air cannot escape from it along the bronchus, it seems reasonable to suppose that fluid cannot leave it by this route. Some mechanism other than

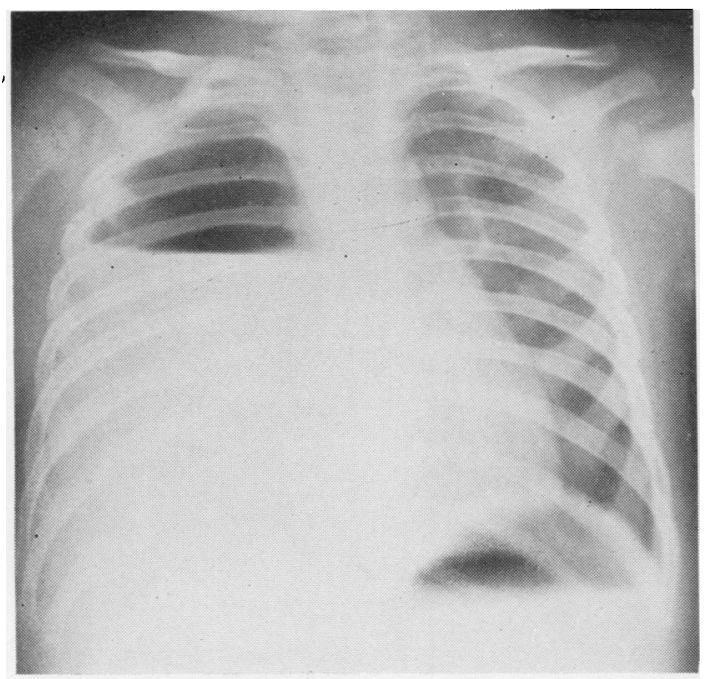

FIG. 3a

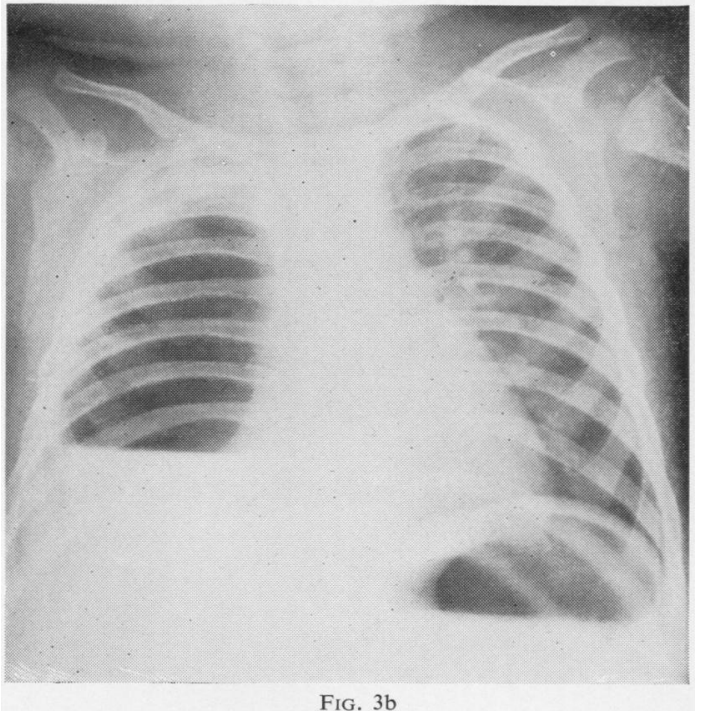

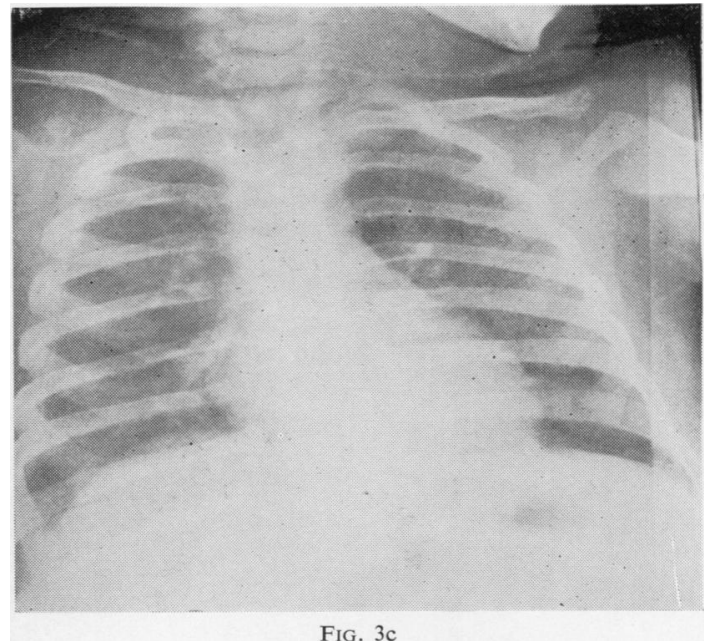

FIG. 3 (a, b, and c).-Radiographs showing spontaneous disappearance of the fluid in a tension pyopneumothorax in a baby aged 9 months.

drainage along the bronchus must be sought to explain the spontaneous emptying of a tension lesion.

The foregoing clinical and radiological observations have certain practical applications in treatment.

\section{Control of the Size of the AIr Space}

An air space, either in the lung or pleura, will not increase in size when an equilibrium is reached between the maximum intrabronchial pressure and the pressure within the space. That such an equilibrium is reached is shown by the fact that when an air space that has remained constant in size is intubated, there is often a persistent escape of air along the tube. The first object is, therefore, to try to establish this equilibrium with the smallest possible volume of air in the space. To do this one has to decrease the intrabronchial pressure and increase the intracavitary pressure if this can be done without increasing the size of the cavity.

To avoid peaks of intrabronchial pressure it is important to keep the baby from crying and from coughing. If he cries lustily, he can rapidly inflate the lesion. One should avoid sticking the baby unnecessarily with needles that not only make him cry but may provoke a bout of coughing. The appearance of a large pneumothorax after aspiration is usually attributed to puncture of the lung, but in many instances may be due to rapid inflation of a small lesion due to the greatly increased bronchial pressure when the baby cries. It is preferable to give an antibiotic that is effective when given by mouth rather than by injection. A good deal can be done by the judicious use of sedatives that may convert grunting expiration to quiet breathing. 


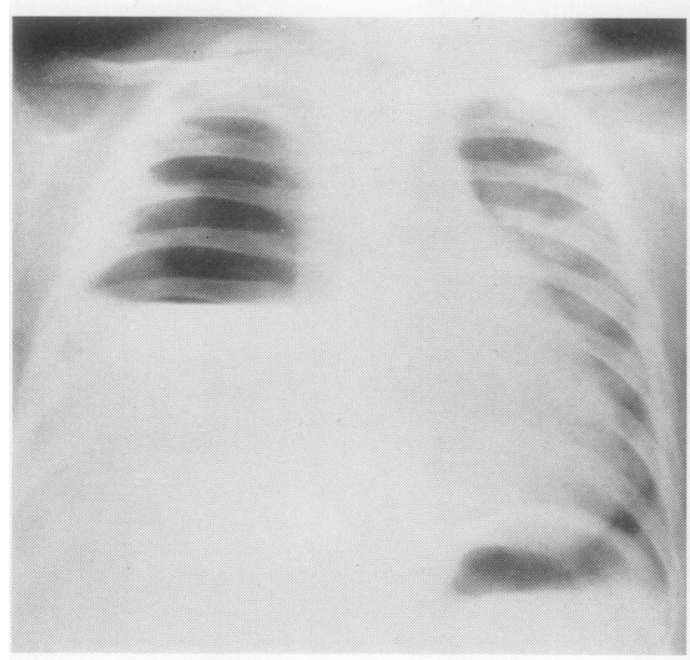

FIG. 4a

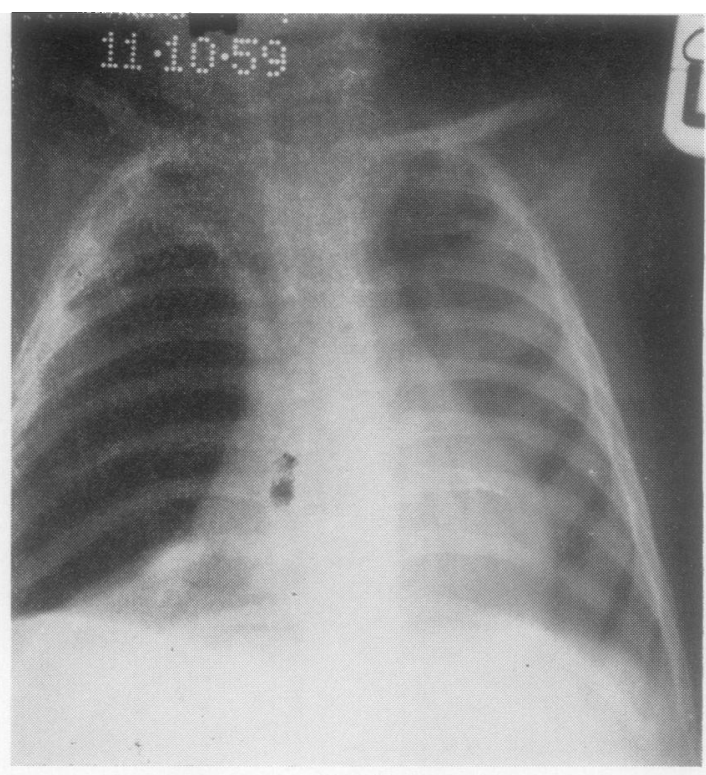

FIG. $4 b$

Fic. 4 ( $a$ and b).-Films showing the spontaneous disappearance of fluid from a tension pyopneumothorax in a baby aged 3 months leaving the characteristic empty space.

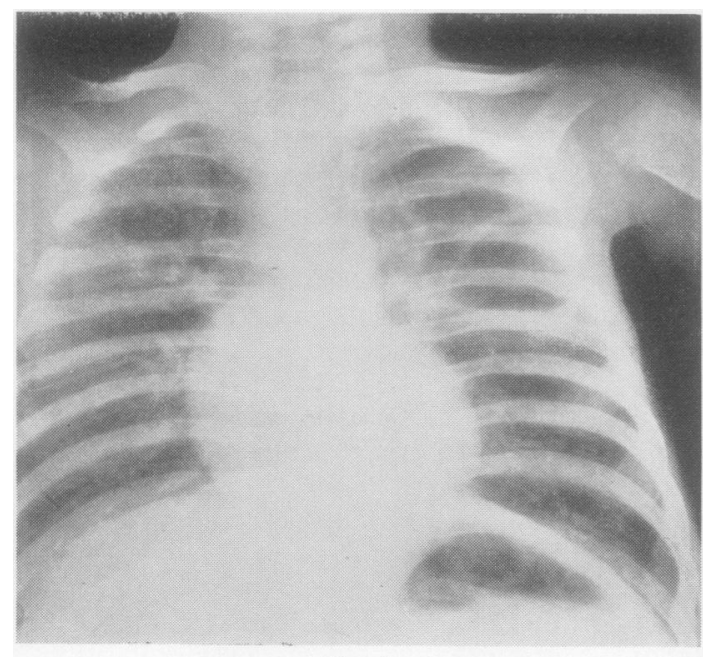

FIG. 5a

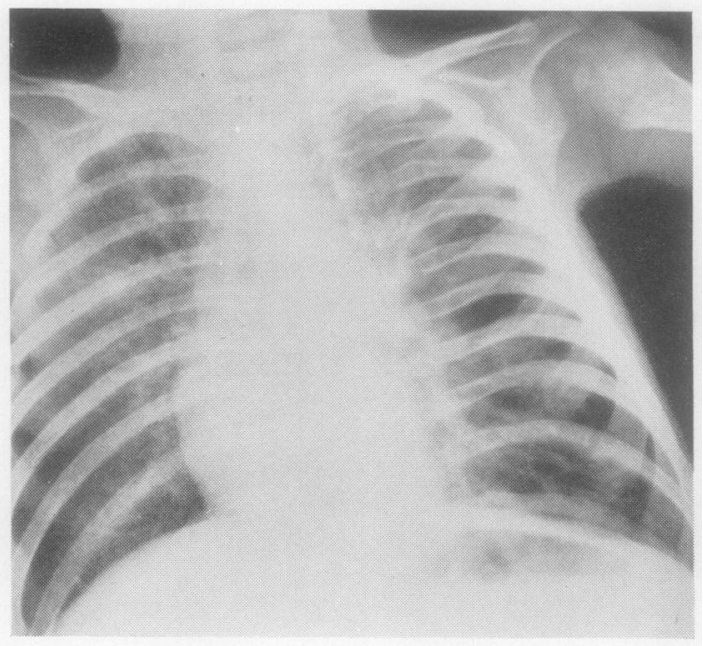

FIG. 5b

Fig. 5 ( $a$ and b). -Films showing disapparance of fluid from a pneumatocele which increases in size as it empties. 


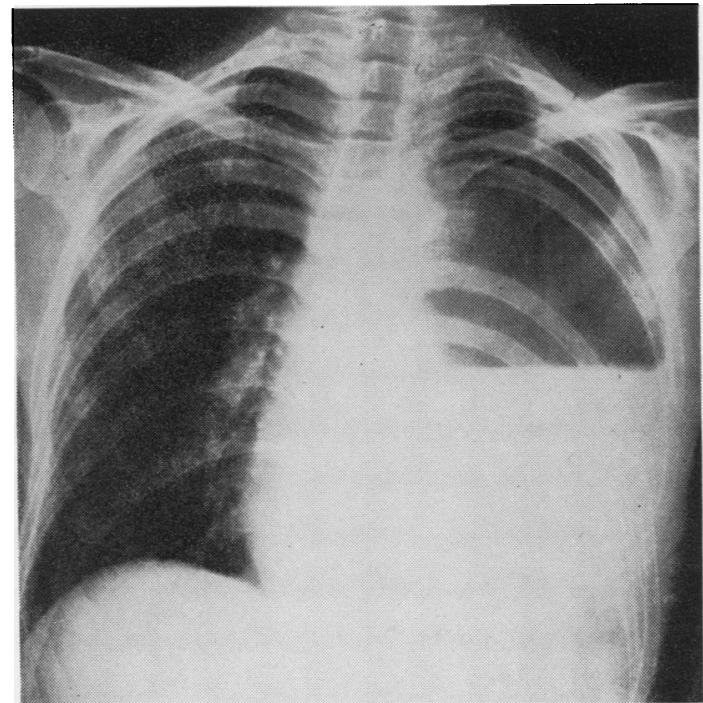

FIG. 6a

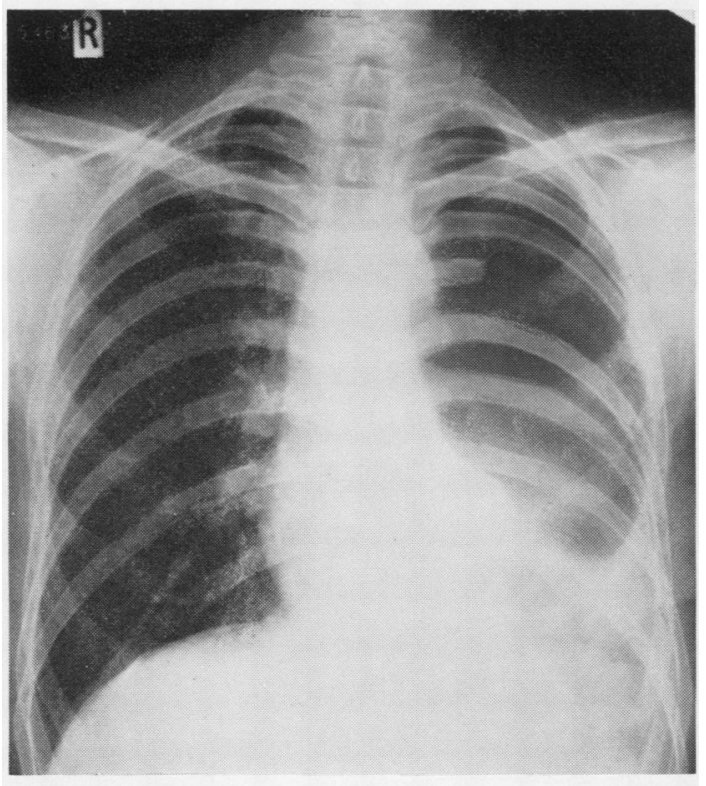

FIG. 6b

FIG .6 ( $\mathrm{a}$ and $\mathrm{b}$ ).--Radiographs demonstrating the effect of positive pressure in a pneumonectomy space. Positive pressure was maintained from the tenth to the seventeenth post-operative day.
It is possible to increase the intracavitary pressure by posture. The baby should be kept lying on the affected side so that the pressure within the space is raised and will more quickly equal the intrabronchial pressure.

\section{Effect of Tension in an Air Space}

Fluid collects in an air space when the exudative or transudative pressure in its wall exceeds the air pressure within it. On the other hand, if the air pressure within the space is high, as in a tension lesion, and exceeds the exudative or transudative pressure in the wall, the fluid disappears by absorption into the wall of the space. An understanding of this mechanism explains the behaviour of the tension lesions of staphylococcal pneumonia, and has a bearing on treatment. If, during the phase of active infection in a staphylococcal pneumonia, the tension lesion empties and remains empty, fibrin is not deposited on its wall. When, thereafter, the infection is overcome by antibiotics, healing will occur without significant pleural thickening. If the intracavitary pressure is reduced by aspiration or drainage, all is well as long as the lung expands quickly. Should the lung fail to expand because of the presence of a bronchopleural fistula, fluid collects in the low-pressure space, and fibrin is deposited on the pleural surface. Fortunately, children up to the

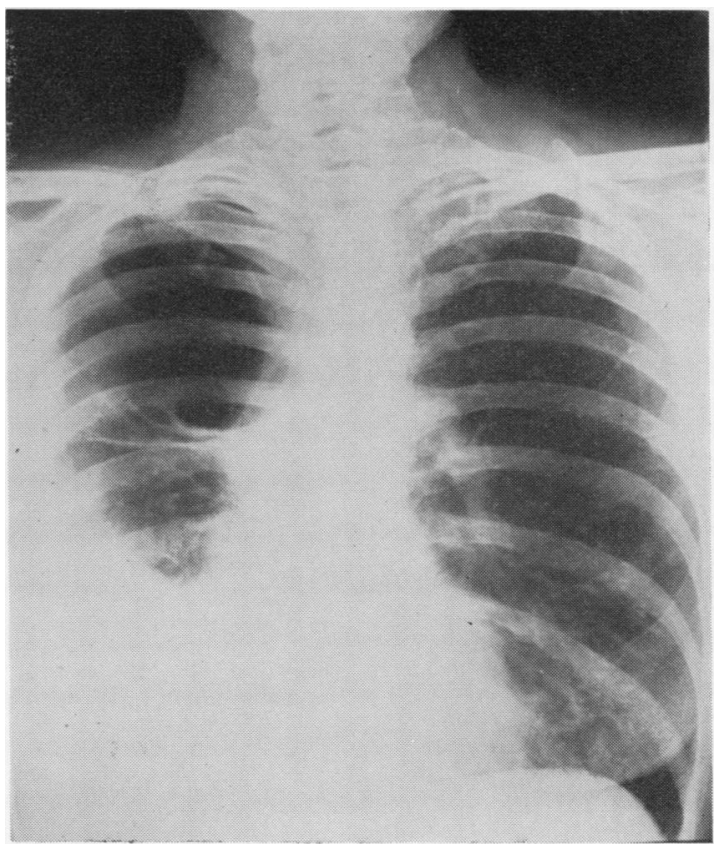

Fig. 7.-An empty tuberculous tension cavity coexistent with a tuberculous pleural effusion. 


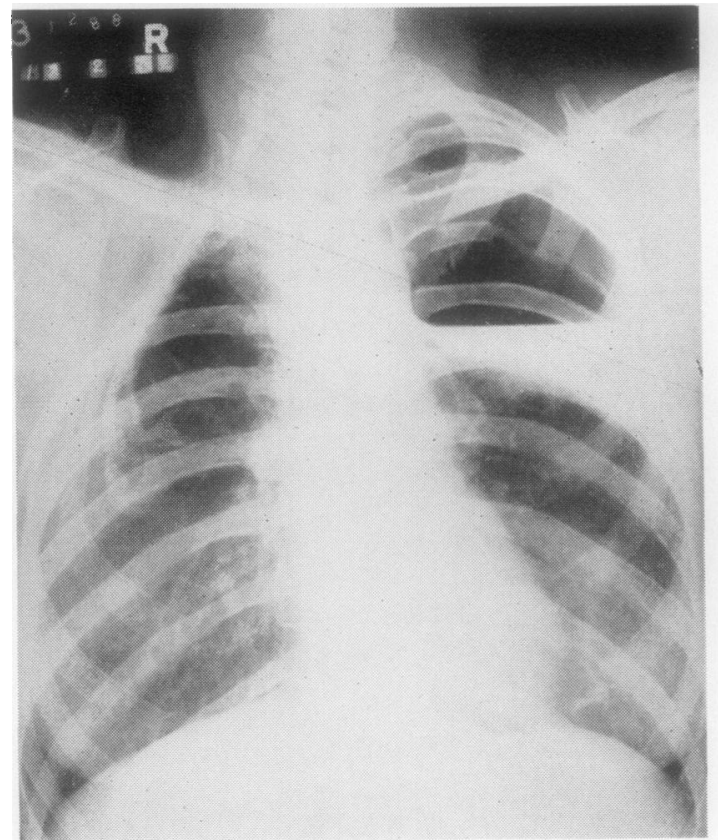

FIG. 8a

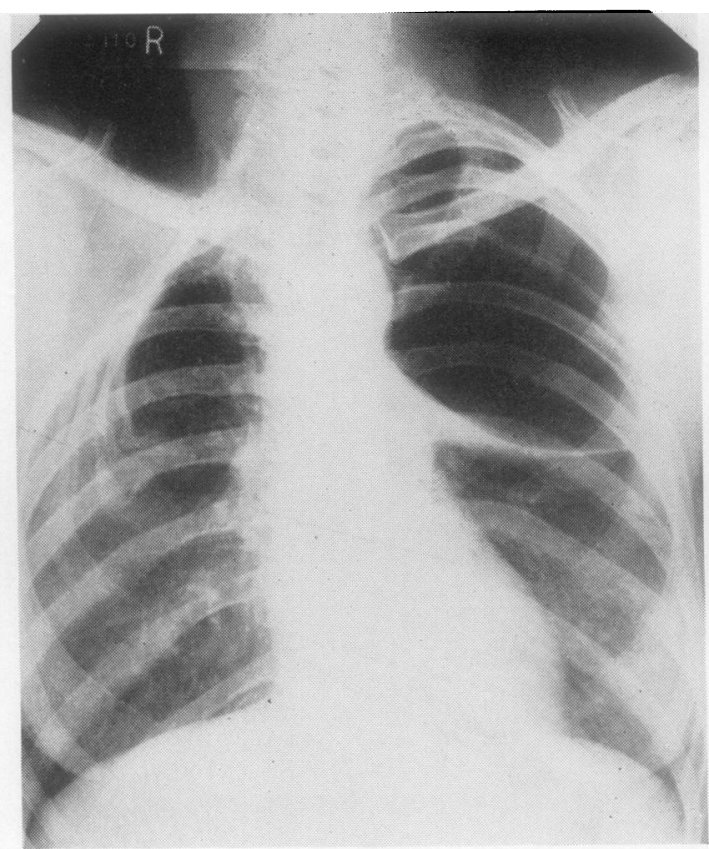

FIG. 8b

FIG. 8 (a and b).-Films showing the accumulation of fluid in an ineffectively filled extrapleural pneumothorax and its disappearance after maintenance of a constant positive pressure.

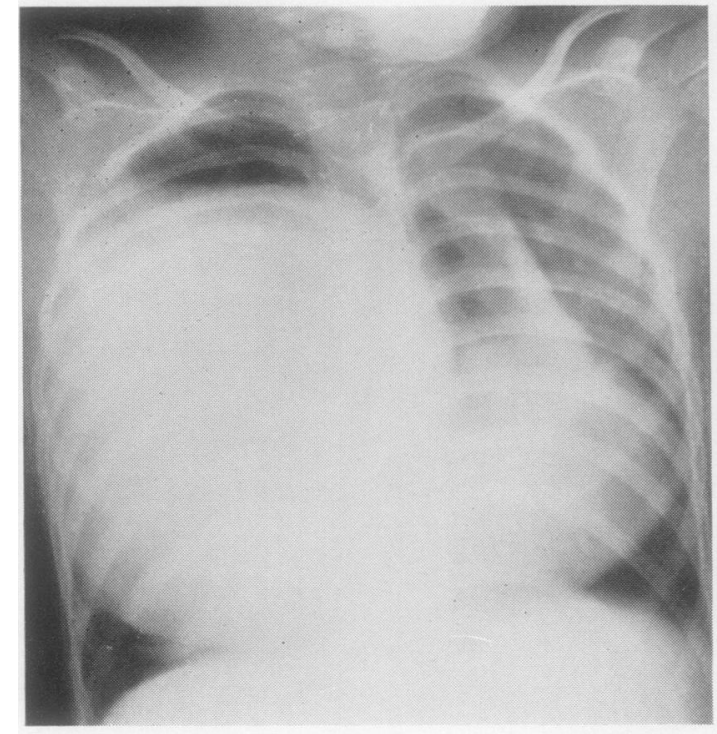

FIG. 9a

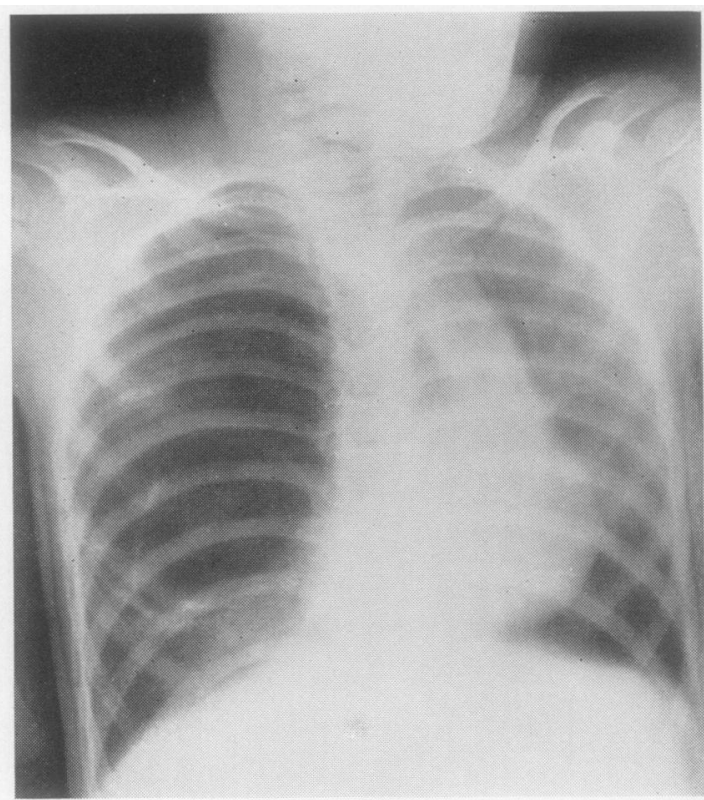

FIG, 9b

Fig. 9 (a and b).-Films illustrating the spontaneous emptying of a solitary tension cyst of lung. 
age of 3 years have a remarkable capacity to absorb inflammatory exudates, but, on occasion, unnecessary and ineffective drainage may give rise to pleural thickening that would not otherwise have occurred.

Relation between Pressure within a Cavity and Capillary Pressure in Cavity Walls

The interrelation of pressure within a cavity and the exudative pressure in the capillaries of the cavity wall is evident in many circumstances other than staphylococcal pneumonia as is illustrated by the following examples.

(1) The Pneumonectomy SPaCe.-If positive pressure is maintained in a pneumonectomy space, the fluid in it disappears. Ten days are allowed to elapse after the operation to ensure sealing of the chest wall incision (Fig. 6a). If air is then introduced daily for a week so as to keep up a positive pressure, the pneumonectomy space becomes completely empty (Fig. 6b).

(2) Tuberculous Tension Cavity of Lung.- A feature of the tuberculous tension cavity is that it is empty. The patient whose case is illustrated here (Fig. 7) had a large empty cavity at the right apex for many months. The pressure in the cavity was $8 / 0 \mathrm{~cm}$. water. There was also a pleural effusion containing many tubercle bacilli. The appearance of fluid in the tension cavity is prevented by the pressure within it.

(3) Extrapleural Pneumothorax. - Accummulation of fluid in an extrapleural pneumothorax depends largely on whether or not a constant positive pressure can be maintained in it. This is illustrated in one patient (Fig. 8a) in whom the same cycle of events was repeated over and over again. When the pneumothorax was filled fortnightly, fluid always collected. When it was filled daily, the fluid could always be made to disappear (Fig. 8b).

(4) GiANT CyST OF LUNG.-In a solitary cyst of lung in a child, all the fluid may disappear within 24 hours without any of it being coughed up. Such an occurrence is illustrated by two films (Fig. 9a and $9 b$ ) of a young girl.

In a hydrocele that is constant in size there is a continuous interchange between the fluid in the sac and the blood in the capillaries of its wall. If methylene blue is injected into the sac of a hydrocele, the dye appears in the urine in about an hour. This suggests that, although there is this interchange of fluid, the relatively indistensible sac causes an equilibrium to be reached between the pressure within the sac and the transudative pressure in its wall. The hydrocele remains constant in size.

The blister of a burn increases in size until the tension maintained by the intact epithelium equals the exudative pressure from the deeper layer. If the epithelium is removed the raw area weeps serum.

\section{Discussion}

The most important factor in the treatment of staphylococcal pneumonia in infancy is the correct use of antibiotics. Conservative treatment does not mean doing nothing apart from giving antibiotics. An essential part of the management is the control of the size of a potentially dangerous pneumatocele or pneumothorax. Expiratory effort against a closed glottis must be avoided. A tracheostomy, while useful in older patients, is difficult to manage in a tiny infant. The spreading surgical emphysema sometimes associated with chest injury or with air leak from the raw surface after segmental lobectomy can be arrested by controlling the raised intrabronchial pressure of expiratory effort. On occasion it is sufficient to sedate the patient. At other times tracheostomy is essential. A baby that has a valvular communication between a bronchus and a pneumatocele or pneumothorax is in a similar situation. Elevation of the intrabronchial pressure due to crying, coughing, or grunting expiration results in progressive inflation of the lesion.

It is well known that the pressure in an artificial pneumothorax is at its highest when measured while the patient is lying on the side of the pneumothorax. If the baby is kept lying on the side of the pneumatocele or pneumothorax, the pressure within the lesion is at its height for a given volume of air in the space, and inflation of the lesion from the bronchus occurs less readily.

After the baby has been protected from the risk of early death due to suffocation, controlled tension within the space, far from causing late morbidity, is an important factor in the dramatic transition from gross pyopneumothorax to normal radiographic appearances which is such a remarkable feature of this disease.

\section{SUMMARY}

The principles of conservative management relating to the control of the size of a pneumatocele or pneumothorax are described.

The effect of tension on the fluid content of an air space is discussed.

I wish to thank Mr. N. R. Barrett for his help in the preparation of this paper, and for allowing me to use the radiographs of his case of solitary cyst of lung. I am indebted also to my colleagues Dr. M. W. Arthurton and Dr. E. Rosenblum.

\section{REFERENCE}

Sabiston, D. C., Hopkins, E. H., Cooke, R. E., and Bennett, I. L. (1959). J. thorac. Cardiovascular Surg., 38, 421. 